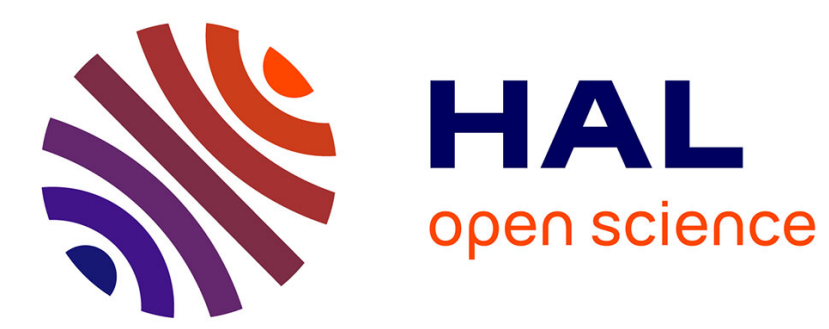

\title{
Socio-histoire du lien entre activité physique et santé de 1960 à 1980
}

\author{
Brice Favier-Ambrosini
}

\section{To cite this version:}

Brice Favier-Ambrosini. Socio-histoire du lien entre activité physique et santé de 1960 à 1980 . Santé Publique, 2016, S1 (HS), 10.3917/spub.160.0013 . hal-01756739

\section{HAL Id: hal-01756739 \\ https://hal.science/hal-01756739}

Submitted on 2 Apr 2018

HAL is a multi-disciplinary open access archive for the deposit and dissemination of scientific research documents, whether they are published or not. The documents may come from teaching and research institutions in France or abroad, or from public or private research centers.
L'archive ouverte pluridisciplinaire HAL, est destinée au dépôt et à la diffusion de documents scientifiques de niveau recherche, publiés ou non, émanant des établissements d'enseignement et de recherche français ou étrangers, des laboratoires publics ou privés. 


\section{SOCIO-HISTOIRE DU LIEN ENTRE ACTIVITÉ PHYSIQUE ET SANTÉ DE 1960 À 1980 \\ Brice Favier-Ambrosini}

S.F.S.P. | « Santé Publique »

2016/HS S1 | pages 13 à 24

ISSN 0995-3914

Article disponible en ligne à l'adresse :

https://www.cairn.info/revue-sante-publique-2016-HS-page-13.htm

\section{Pour citer cet article :}

Brice Favier-Ambrosini, "Socio-histoire du lien entre activité physique et santé de 1960 à 1980 », Santé Publique 2016/HS (S1), p. 13-24.

DOI 10.3917/spub.160.0013

Distribution électronique Cairn.info pour S.F.S.P..

(C) S.F.S.P.. Tous droits réservés pour tous pays.

La reproduction ou représentation de cet article, notamment par photocopie, n'est autorisée que dans les limites des conditions générales d'utilisation du site ou, le cas échéant, des conditions générales de la licence souscrite par votre établissement. Toute autre reproduction ou représentation, en tout ou partie, sous quelque forme et de quelque manière que ce soit, est interdite sauf accord préalable et écrit de l'éditeur, en dehors des cas prévus par la législation en vigueur en France. Il est précisé que son stockage dans une base de données est également interdit. 


\section{$\mathbf{1}^{\text {re }}$ partie - L'activité physique dans les politiques publiques}





\title{
Socio-histoire du lien entre activité physique et santé de 1960 à 1980
}

\section{Social history of the relationship between physical activity and health from 1960 to 1980}

\author{
Brice Favier-Ambrosini ${ }^{1}$
}

\section{$\hookrightarrow \rightarrow$ Résumé}

Objectif : Nous inscrivant dans une sociologie pragmatique de l'étude des problèmes de santé publique, nous nous attachons à éclaircir l'émergence et la constitution du discours amenant à considérer l'Activité Physique et Sportive (APS) comme facteur de santé pour le plus grand nombre dans la période 1960-1980.

Méthodes : Notre travail se base sur l'étude de trois corpus. Le premier est constitué des articles de la revue La Santé de l'Homme depuis 1950 qui traitent de la question de l'APS comme facteur de santé. Le deuxième se compose de l'ensemble des thèses françaises produites autour de cette question depuis 1940. Le troisième représente un corpus hétérogène, sur la base de renvois intertextuels, constitué d'études scientifiques, de textes de cadrage des politiques de santé ainsi que d'ouvrages de vulgarisation fréquemment cités et repris autour de cette thématique.

Résultats : La trajectoire de la question de l'APS comme facteur de santé subit une inflexion au milieu des années 1960 par un dé-confinement depuis l'arène scientifique pour être portée sur le devant de la scène publique en lien avec la gestion du problème des maladies cardiovasculaires. Dans le cadre d'un paradigme épidémiologique où sont identifiés des facteurs de risque susceptibles d'augmenter la probabilité de contracter ce type de pathologies, la sphère médicale va travailler à rendre tangible l'idée selon laquelle l'APS est une solution légitime pour répondre à ce problème. En France, ce sont les cardiologues de la Fédération Française de Cardiologie qui vont s'inscrire comme les détenteurs légitimes du problème.

Mots-clés: Maladies cardiovasculaires; Santé publique ; Sports.

\section{$\sqsubset \rightarrow$ Summary}

Objective: Based on a pragmatic sociology approach to the study of public health problems, we tried to clarify the emergence and constitution of the discourse that considers Sport and Physical Activity (SPA) to be a positive health factor for the majority of the population during the period 1960-1980.

Methods: This study was based on the three bodies of knowledge. The first was composed of articles published in La Santé de l'Homme since 1950, dealing with the issue of SPA as a health factor. The second was composed of all French doctorate theses on this issue since 1940 and the third consisted of a heterogeneous body of intertextual references, consisting of scientific studies, health policy texts and popular books frequently cited on this topic.

Results: The trajectory of SPA as a health factor underwent a major change in the mid-1960s, by emerging from the strictly scientific field to become a general public approach to the prevention of cardiovascular disease. In the context of an epidemiological paradigm comprising risk factors likely to increase the probability of developing this type of disease, the medical community worked to enhance the practical application of the idea that SPA is a legitimate solution to address this issue. In France, cardiologists of the French Federation of Cardiology became the legitimate leaders in this field.

Keywords: Cardiovascular diseases; Public health; Sports.

\footnotetext{
${ }^{1}$ Université Paris-Est Marne-La-Vallée Laboratoire ACP. EA 3350 - ACP - Bois de l’Étang 5, Boulevard Descartes - 77454 Marne-la-Vallée. 


\section{Introduction}

La pratique des activités physiques (AP) dans un but de préservation et de restauration de la santé des individus est un fait largement attesté depuis l'antiquité [1]. Depuis Aristote, conseillant la gymnastique pour la santé en évitant les excès, en passant par la méthode d'éducation physique à visée hygiéniste de la Ligue Française d'Éducation Physique (LFEP) [2], « c'est une certitude, l'activité physique contribue largementà préserver et à améliorer la santé » [3] . Ce lien entre AP et santé semble donc ancien ; il est le fruit de travaux scientifiques (comme par exemple les travaux des médecins hygiénistes du $19^{\mathrm{e}}$ siècle, tels que Ferdinand Lagrange), de représentations sociales [4] mais il est aussi mobilisé comme un slogan de nature politique servant différentes institutions. Pour autant l'impact du sport proprement dit $^{1}$ sur la santé a été largement discuté et contesté depuis son émergence au milieu du $19^{\mathrm{e}}$ siècle. D'abord regardé avec méfiance, ce n'est qu'assez tardivement qu'il a pu être pris en compte comme un outil important au service de la santé [1]. Au final ce n'est véritablement qu'à partir des années 1960 qu'un champ épistémique commence à se solidifier autour de la relation sport-santé et que la sphère des politiques publiques va véritablement intégrer l'Activité Physique et Sportive (APS) comme enjeu central dans la prévention de maladies dites de «civilisation » [5]. Si un certain nombre de travaux socio-historiques ont étudié l'émergence et la diffusion du rôle de l'APS dans la prévention de certaines pathologies particulières telles que l'obésité [6], le VIH [7], le diabète [8], la mucoviscidose [9], les maladies cardiovasculaires [10]... nous souhaitons pour notre part travailler à identifier la constitution de cette thématique globale en tant que « cause » [11] c'est-à-dire en tant que thématique socialement et politiquement mobilisatrice.

\section{Méthodes}

Nous inscrivant dans une sociologie pragmatique de l'étude des problèmes de santé publique [12], nous nous attachons à repérer la manière dont certains acteurs vont promouvoir l'APS comme facteur de santé. Nous serons ainsi attentifs aux opportunités que saisiront ces acteurs

${ }^{1}$ Entendu comme une activité à visée compétitive et performative. pour s'installer comme "propriétaires légitimes du problème » [13] dans l'espace concurrentiel que représente le champ du « sport-santé » au début des années 1960 ainsi qu'à leurs stratégies argumentatives.

Pour cela, nous avons tout d'abord constitué un corpus systématique en parcourant l'ensemble des articles de la revue La Santé de l'Homme éditée par le Centre National d'Éducation Sanitaire Démographique et Sociale (CNESDS) puis par le Comité Français d'Éducation pour la Santé (CFES) et enfin l'Institut National de Prévention et d'Éducation pour la Santé (INPES) ${ }^{2}$ depuis 1950, afin de retracer la trajectoire socio-historique de l'APS comme facteur de santé dans le champ de l'éducation sanitaire à destination du grand public. Nous considérons l'étude de ce corpus comme un des indicateurs permettant de mettre en évidence l'émergence et l'importance que prend cette thématique dans l'arène publique et sa constitution en tant que cause socialement et politiquement mobilisatrice. Ont été retenu et quantifié les articles où la question de l'APS en lien avec la santé est évoquée dans l'argumentaire, que ce soit de manière centrale ou secondaire. Concernant les modalités de traitement des données, nous avons été attentifs aux différentes thématiques investies par les auteurs argumentant autour de la place et du rôle de l'APS dans un objectif de santé ; aux types de savoirs mobilisés dans leur discours ainsi qu'à leur statut professionnel quand les articles étaient signés. Par ailleurs, nous avons recensé l'ensemble des thèses françaises produites autour de cette question depuis 1940 afin d'appréhender, en partie, son évolution dans la sphère scientifique. Ici nous avons retenu et quantifié, à la fois les thèses centrées spécifiquement sur le lien entre APS et santé (par exemple Duval, 1950 et Brondani, 1974) ${ }^{3}$ et celles faisant simplement une place à cette thématique, mais dont l'objet central est autre (par exemple Kahn, 1974 et Zarka, 1981). D'autre part, nous avons constitué un corpus hétérogène, sur la base de renvois intertextuels, constitué d'études scientifiques marquantes, de texte de cadrage de politiques de santé et d'ouvrages de vulgarisation fréquemment cités et repris concernant cette thématique afin d'appréhender le processus de dé-confinement depuis l'arène scientifique vers le grand public.

\footnotetext{
${ }^{2}$ Le CFES remplace le CNESDS en 1972. Le CFES est remplacé par l'INPES en 2002.

${ }^{3}$ Les références entre parenthèses correspondent aux éléments de notre corpus que nous avons listés en annexe. Les références bibliographiques n'appartenant pas au corpus sont quant à elles numérotées en chiffres arabes etfigurent en fin d'article dans la rubrique « Références ».
} 


\section{Résultats}

\section{La question de l'APS dans un but de santé subit une publicisation importante à partir des années 1960}

L'ensemble des indicateurs analysés nous amène à considérer que la trajectoire de la question de l'APS comme facteur de santé prend une inflexion particulière au milieu des années 1960 pour être mise sur le devant de la scène publique et diffusée au plus grand nombre.

En effet, si la sphère de l'Éducation Physique et Sportive (EPS) travaille depuis le début du siècle à la promotion de l'AP (pensée comme une EP méthodique et rationnelle) pour préserver et améliorer la santé des élèves, notamment autour des travaux du Dr Tissié [14] et de la LFEP, peu de propositions sont faites quant à l'éducation physique de l'adulte en général dans un souci de santé publique [15] avant le milieu des années 1960, notamment de la part du corps médical. Ainsi concernant le champ de l'éducation pour la santé, Radel [6] montre que les films à vocation sanitaire édités par les différentes structures qui s'investissent dans ce domaine et qui se développent à partir des années 1950 témoignent très clairement d'un faible intérêt pour l'APS avant le milieu des années 1970. L'étude systématique des articles travaillant la question des liens entre APS et santé de manière centrale ou secondaire dans la revue La santé de l'Homme va dans le même sens. Leur nombre reste assez faible jusqu'au début des années 1970 puis celui-ci augmente drastiquement. Nous recensons ainsi 43 articles évoquant cette relation entre 1960 et 1970 , ce chiffre atteignant 73 pour la décennie 1970-1980 puis 127 entre 1980 et 1990. Le nombre d'articles centrés spécifiquement sur la relation APS-santé est également révélateur : de 27 articles entre 1960 et 1972, ce chiffre passe à 50 entre 1973 et 1983, puis à 73 entre 1984 et 1993 . D'autre part, l'évolution du nombre de thèses françaises faisant une place à cette thématique est également frappante. Nous avons en effet relevé 64 thèses soutenues entre 1940 et 1950, 101 entre 1950 et 1960, ce chiffre s'élevant à 137 entre 1960 et 1970 pour atteindre 461 dans la décennie 1970. L'idée que l'APS puisse être un outil de santé publique semble donc se diffuser massivement auprès des médecins français (la quasi-totalité des thèses relevées sont des thèses de médecine) à partir du milieu des années 1960 et dans la décennie 1970. Cependant, cette question commence à être grandement travaillée dès le début des années 1950 dans l'arène scientifique internationale. Ainsi, en 1953 Morris et al., (Morris et al., 1953) dans le cadre de l'épidémiologie des pathologies cardiaques des travailleurs, émet l'hypothèse selon laquelle l'AP pourrait jouer un rôle dans la pathogénèse des cardiopathies ischémiques ouvrant tout un champ de recherche autour de la question de l'AP comme outil de santé publique (Brown et al., 1957 ; Breslow et al., 1960). Ainsi, une communauté épistémique travaillant cette thématique s'ouvre véritablement dans l'arène scientifique internationale au début des années 1950 par l'intermédiaire d'une série d'études en épidémiologie des pathologies cardiaques des travailleurs.

Au final, l'ensemble de ces indicateurs amène à mettre en évidence, un certain confinement de la question de l'AP comme facteur de santé publique dans l'arène scientifique, ne subissant pas une diffusion importante auprès du grand public avant le milieu des années 1960.

\section{0-1970 : I'ouverture d'une fenêtre d'opportunité pour la promotion de l'APS comme facteur de santé au travers de la prise en charge des maladies cardiovasculaires}

Si le rôle de l'APS comme facteur de santé ne subit pas de publicisation massive jusqu'à la fin des années 1960, une première inflexion de sa trajectoire s'organise à partir de cette période. Plus précisément, dans les sphères médiatiques, politiques et scientifiques, la promotion large de la pratique de l'APS dans un objectif sanitaire s'inscrit dans la construction du problème de santé publique que représentent les maladies cardiovasculaires (MCV) à cette époque. Les promoteurs du sport-santé (entendu au sens large) s'appuient en effet sur le fait que les années 1960 sont marquées par une bascule quant aux problèmes sanitaires majeurs où les préoccupations liées aux maladies infectieuses (tuberculose, poliomyélite...) diminuent pour laisser progressivement la place aux maladies métaboliques et dégénératives et à ce qui va devenir « le fléau $n^{\circ} 1$ " (Froment, 1967) à savoir les MCV et plus particulièrement l'athérosclérose. Ainsi, sont par exemple mobilisées dans un rapport du Conseil de la Coopération Culturelle du Conseil de l’Europe spécifiquement dédié à la problématique du sport-santé (Réville, 1970), les statistiques internationales de l'Organisation Mondiale de la Santé du milieu des années 1960 qui montrent que les principales causes de décès, dans les pays industrialisés, sont les maladies du cœur et des vaisseaux et les accidents vasculaires cérébraux qui, additionnés, sont responsables de $45 \%$ des décès (OMS, 1967). Loin de se cantonner à un phénomène international éloigné des problématiques françaises, le cas des MCV va progressivement être identifié comme un véritable fléau national. Ainsi dès 1960, la sphère de l'éducation à la 
santé, par l'intermédiaire du CNESDS fait de « la prévention des maladies chroniques et notamment des affections cardiovasculaires » (Delore, 1960) un de ses objectifs prioritaires. Son organe de diffusion, la revue La santé de l'Homme multiplie ainsi dès les années 1960 et de manière exponentielle le nombre d'articles et numéros spéciaux, amenant à considérer une prévalence importante d'un certain nombre de maladies dites de " civilisation », c'està-dire liées au style de vie moderne, dont l'ensemble des pathologies cardiaques en sont la figure de proue (Calo, 1960) et qui en 1964 représentent $44 \%$ de l'ensemble des causes de décès en France (Aujoulat, 1964). Ce chiffre ne fera que progresser amenant, entre autres, les membres de l'Académie de médecine (Leclainche, 1967) à porter ce problème sur le devant de la scène et faire pression auprès des pouvoirs publics afin de voir ces pathologies, notamment l'athérosclérose, reconnues comme un véritable « problème public » nécessitant une attention prioritaire de l'État (Froment, 1967). L'argumentaire s'appuie ainsi sur une montée statistique des taux de mortalité et de morbidité mais également sur des préoccupations économiques dans la mesure où ces affections impactent la collectivité par un coût important (Perrin, 1967). Ainsi, la lutte contre les affections cardiovasculaires est définitivement mise à l'agenda politique français à partir de 1970 dans les principales options du sixième plan de Santé (Journal officiel du 10 juillet 1970). Au niveau international, l'OMS fait par exemple de l'année 1972 «l'année du cœur». Les années 1960-1970 sont ainsi marquées par le dé-confinement du problème des maladies cardiovasculaires qui accède à l'espace public et devient une préoccupation centrale des pouvoirs publics.

D'autre part, en suivant Gilbert et Henry [12], l'émergence d'un problème de santé publique est avant tout le résultat de processus de mobilisation et d'action collective qui ont notamment pour enjeu la définition du problème lui-même et qui contribuera à légitimer ou non le cours de l'action publique tel qu'il se déroule. En ce sens, le problème des MCV va être défini et construit par la sphère médicale de manière singulière, sur la base d'un paradigme épidémiologique alors en plein développement à cette période [16] conduisant à légitimer certaines solutions. De grandes études prospectives de cohortes sont ainsi mises en place, aux États-Unis au tournant des années 1950 dans une perspective d'épidémiologie cardiovasculaire et vont permettre de préciser la définition du problème en identifiant un certain nombre de facteurs susceptibles d'augmenter la probabilité de développer une pathologie cardiaque. À ce titre, l'enquête de Framingham, qui commence en 1947 dans le cadre de l'US Public Health
Service (USPHS) dont les premiers résultats sont diffusés partir des années 1960 (Dawber et al., 1964) s'installera comme une étude «exemplar» [17] dans le sens où elle sera fortement relayée par l'ensemble des publications postérieures et influencera massivement la compréhension du problème des $\mathrm{MCV}^{4}$. Cette étude, tout comme celle des «sept pays » en Europe (Keys, 1970), va ainsi faire de la notion de facteur de risque « un nouveau cadre paradigmique » [21] pour penser la maladie et sa prise en charge par le milieu médical, et s'imposer comme un schéma conceptuel hégémonique, marquant fortement l'éducation pour la santé en France [22]. Est ainsi repéré et diffusé, par le CNESDS puis le CFES, le rôle néfaste de certains facteurs quant à la probabilité de développer l'athérosclérose : le tabac, l'hypertension artérielle, le diabète, l'obésité, la sédentarité... (Aujoulat, 1972). Comme le souligne Cazal [10], dans un contexte sociétal où le risque prend une part de plus en plus considérable dans les modes de pensée et d'action [23], «la statistique permet de quantifier, d'ordonner le réel et structure le mode d'intervention des politiques de santé publique et de recherche médicale ». Dit autrement, « l'approche des facteurs de risque » permet de délimiter clairement « ce qui fait problème » et ainsi justifier une prise en charge par l'institution. En définissant le problème de cette manière, la sphère médicale étend sa légitimité sur les comportements susceptibles d'atténuer les pathologies qui produisent l'athérosclérose conduisant ainsi à une "sanitarisation du social» [24]. Au final, la période 1960-1970 est caractérisée par l'identification et la construction du problème des MCV en termes de facteurs de risque susceptibles de les développer. Dans ce cadre, le manque d'AP est dénoncé ouvrant ainsi une « fenêtre d'opportunité » pour certains acteurs qui vont militer pour une reconnaissance de l'APS comme facteur de santé. La trajectoire de cette question subit ainsi à partir de ce moment, une véritable inflexion et est mise sur le devant de la scène publique.

\section{Promouvoir l'APS pour prévenir les maladies cardiovasculaires en profitant d'une fenêtre d'opportunité et en comblant la faiblesse des assertions scientifiques}

C'est en s'appuyant sur le cadrage du problème des MCV sur la base d'un paradigme épidémiologique en termes de facteurs de risque, que la sphère médicale construit, tout en se l'appropriant, l'idée selon laquelle la pratique des AP

\footnotetext{
${ }^{4}$ Pour une étude historique de la constitution de cette enquête épidémiologique et de ses multiples redéfinitions [11, 19, 20, 21].
} 
est indispensable pour lutter contre ces maladies. La corporation des médecins est en effet hégémonique sur la question à cette période comme en témoignent, d'une part, le fait que l'immense majorité des thèses françaises produites sur la question pour la période 1960-1985 sont des travaux d'exercice en médecine, pharmacie et odontologie, et d'autre part que la quasi-totalité des articles diffusés sur cette problématique par La santé de l'Homme émanent de cette corporation ${ }^{5}$. En identifiant certains modes de vie potentiellement favorables à la survenue d'une maladie cardiaque, il en est alors déduit que la pratique d'une AP est une solution au problème des pathologies cardiovasculaires. En témoigne, au niveau national, le nombre d'articles travaillant la relation APS-santé dans cette perspective qui croît de manière exponentielle dans la revue La Santé de l'Homme à partir de la fin des années 1960 ainsi que les premiers textes de cadrage des politiques publiques du "Sport pour Tous » au niveau européen qui insistent sur cette thématique (Astrand, 1969; Réville, 1970), ou le nombre important de thèses françaises travaillant spécifiquement la relation APS-MCV à partir du milieu des années 1970. Plus précisément nous avons relevé dix articles traitant spécifiquement ou en partie cette thématique entre 1960 et 1970, soit $21 \%$ de l'ensemble des articles évoquant la relation APS-Santé. Ce chiffre monte à 30 pour la période 1971-1981, soit $40 \%$ des articles traitant du lien entre APS et santé pour passer à 146 entre 1982 et 1992 ce qui représente $30 \%$ de la totalité des articles APS-santé. Les grandes études épidémiologiques vont ainsi permettre de dénoncer les conséquences néfastes de la sédentarité qui va devenir un facteur de risque à part entière et ainsi rendre le raisonnement extrêmement clair : pour prévenir les MCV il s'agit de promouvoir l'AP comme comportement bénéfique pour sa santé. À ce titre, l'analyse du discours médical permet d'éclairer la manière dont ces acteurs vont construire cette idée. Tout d'abord, dans la majorité des cas, le fait qu'aucune étude ne témoigne, par un travail longitudinal, que la pratique d'une AP permet effectivement de prévenir l'athérosclérose, n'est mentionné et ne s'impose pas comme une limite à la promotion de la pratique d'une APS dans ce cadre. En effet, les travaux épidémiologiques exemplars sont essentiellement descriptifs, la sédentarité étant identifiée comme facteur de risque, et non "expérimentaux » où l'on cherche à vérifier que la suppression, ou du moins l'atténuation, des facteurs précédemment individualisés a pour conséquence la diminution du risque impliqué (Milon

\footnotetext{
${ }^{5}$ Sur les 99 articles signés travaillant spécifiquement ou en partie la thématique AP-Santé entre 1960 et 1980, 79 sont écrits par des médecins.
}

et Froment, 1971). La plupart des études de référence sont ainsi transversales et rétrospectives; dans celles-ci sont comparées des populations « actives » (travailleurs actifs le plus souvent) à des sujets inactifs, ce qui laisse des incertitudes quant à l'intervention d'autres variables non contrôlées (par exemple les études de Morris et al., 1953 ou Chapman et al., 1957 qui sont fréquemment relayées). Par ailleurs ces études, produites en grand nombre dans les années 1950-1970, laissent place à certains biais méthodologiques qui viennent affaiblir la robustesse des assertions : imprécision des questionnaires, légèreté de la différence entre métiers physiquement actifs et inactifs, problème des changements de profession... Ainsi, la solidité épistémique des études sur lesquelles s'appuient les promoteurs de l'APS comme facteur de santé, reste faible mais ces écueils sont, dans la majorité des cas passés sous silence. C'est en comblant cette faiblesse qualitative par un faisceau important d'études de natures différentes que les acteurs vont renforcer la robustesse de leur argumentaire et contribuer à le rendre tangible. En effet, sont multipliées les références aux études rétrospectives, transversales et descriptives qui laissent à penser un rôle positif de l'AP sur la prévention des MCV, et ceci dans des orientations variées. Ainsi, si de nombreuses études montrent la moindre prévalence d'infections coronariennes et d'infarctus chez les sujets adultes actifs, des écrits s'intéressant aux personnes âgées sportives ou anciennement sportives sont également utilisés (Par exemple Rook, 1954 ; Karnoven et al., 1956 ; Longueville et al., 1965 ; Diaz et al., 1973). La sphère médicale convoque également systématiquement les travaux en médecine sportive scandinaves, allemands, américains et français étudiant les retentissements physiologiques de programmes d'entraînements chez les sujets sportifs et chez les sujets sédentaires (Saltin et Grimby, 1966; Saltin et al., 1968; Kilbom et al., 1969) afin de chiffrer les bénéfices que peut apporter l'APS sur le fonctionnement physiologique de l'organisme. Enfin, pour renforcer l'argumentaire et pallier ses failles, les études qui témoignent de l'aspect «thérapeutique » de l'AP sur les MCV dès 1970 sont également largement convoquées (Katz, 1967 ; Hollmann et al., 1965 ; Varnauskas et al., 1966). Ainsi en multipliant les références à des études scientifiques travaillant différentes facettes de la relation APS-santé, les acteurs comblent les faiblesses qualitatives des travaux pris isolément. Au final, du point de vue de l'argumentation, c'est la question de la tangibilité des faits qui est ici travaillée. Il s'agit en effet, pour les acteurs, de rendre tangible l'idée selon laquelle l'AP peut être bénéfique pour la prévention et la thérapeutique des MCV. En suivant Chateauraynaud [11], la notion de tangibilité renvoie à la manière dont les acteurs tentent de 
réduire l'incertitude de leur discours en recourant à différentes formes d'administration de la preuve. L'une d'entre elle consiste à « exprimer la consistance du recoupement de multiples versions. [...] Il ne s'agit pas seulement de recouper des témoignages mais de croiser des sources indépendantes, de façon à établir une convergence de vues ». Consciente des faiblesses méthodologiques et épistémologiques des études rétrospectives et transversales qui servent majoritairement à l'argumentation, c'est sur cette modalité d'administration de la preuve que la sphère médicale travaille à rendre tangible l'idée selon laquelle la pratique régulière d'une AP permet de réduire le risque de développer une pathologie cardiaque. La faiblesse probatoire est comblée par une recherche de convergences d'études émanant de perspectives diverses. Au final, malgré les incertitudes, l'argumentaire est rendu suffisamment robuste pour que dès le début des années 1970, l'AP soit promue massivement comme une solution au problème de santé que représentent les MCV.

\section{Un dépositaire légitime du problème en France : La Fédération Française de Cardiologie (FFC)}

Dans l'espace que représente le domaine du sport-santé naissant au début des années 1970, un protagoniste, la FFC, va devenir omniprésent et s'installer comme l'acteur central et légitime portant cette cause dans la période considérée [5]. En étant l'un des seuls groupes sociaux à faire des recommandations pratiques en termes d'APS pour le grand public dans une perspective sanitaire et à les diffuser largement, la sphère des médecins-cardiologues va occuper une place de choix dans ce dossier. En effet, si le raisonnement épidémiologique permet d'envisager l'APS comme facteur de prévention des MCV, encore faut-il pouvoir outiller cette relation par des recommandations pratiques. Or, peu d'indications sont diffusées avant le milieu des années 1970 au grand public comme en témoignent les articles de La santé de l'Homme qui, à cette période, relaient l'idée selon laquelle la sédentarité est un facteur de risque de contracter une pathologie cardiaque et encouragent à la pratique d'une AP mais sans véritablement donner de conseils précis de pratique. Le nombre de ces articles augmente à partir des années 1970 pendant lesquelles certains cardiologues vont multiplier les écrits et diffuser l'idée de l'importance pour les populations de pratiquer une $\mathrm{AP}$, à la fois dans un but de prévention des $M C V$, mais également dans une perspective de réadaptation cardiaque. Se diffuse alors l'idée, controversée dans les années 1960, que certains patients atteints d'une maladie cardiaque peuvent et doivent pratiquer une AP pour améliorer leur rééducation et prévenir une éventuelle récidive, suite à la diffusion des travaux pionniers de Broustet, Bricaud, Emeriau (1976) entre autres. Ces auteurs, membres de la FFC mettent en place les principes d'une rééducation des coronariens par l'AP et vont produire, à partir du milieu des années 1970, un ensemble de documents préconisant certaines activités sportives en fonction des profils de santé qui vont être généralisés à l'ensemble de la population. Ces outils tels que le tableau de Broustet (Souyris-Rolland, 1986) ${ }^{6}$, seront parmi les seuls permettant une opérationnalisation pratique des conseils en termes de « sport-santé » et largement diffusé auprès des populations $[5]^{7}$. Dans ce cadre, les médecins-cardiologues imposent un discours, très repris, qui tend à faire une distinction entre le sport entendu comme activité de compétition où «le dépassement de soi » [25] est recherché et l'AP, où « la juste mesure » [25] fait référence, avec une grande méfiance pour ce premier (Desmare, 1976). Sur la base de ce paradigme de la modération, les médecins-cardiologues recommandent de manière unanime des efforts « d'endurance » (exercices longs et peu intenses par opposition aux efforts dits de « résistance » dont les exercices sont brefs et intensifs), susceptibles d'avoir un rôle préventif quant aux $\mathrm{MCV}^{8}$. Certaines activités dites « d'endurance » ou « d'entretien » vont alors être particulièrement recommandées (marche, vélo, natation, course, jogging, ski de fond) à la fois chez l'enfant et l'adulte (Soisson, 1980 ; Perie et al., 1973). Par ailleurs, que ce soit en prévention primaire et surtout en prévention tertiaire, la gymnastique d'entretien est très valorisée notamment pour les patients atteints de maladies coronariennes en réadaptation. De plus, des recommandations en termes de durée et de fréquence d'exercice sont également délivrées par la FFC. Par exemple, dans le « Guide du cœur et de l'effort physique » (Souyris-Rolland, 1986), il est recommandé de pratiquer trois fois par semaine pendant des séances de trente minutes en recherchant des efforts en endurance. Ce discours à orientation médicale va

\footnotetext{
${ }^{6}$ Dans ce tableau, présenté comme «le meilleur baromètre qui existe actuellement pour guider les sportifs amateurs » (p.91), l'auteur classe les APS susceptibles d'être les plus favorables pour la santé des pratiquants et en déconseille la pratique de certaines.

${ }^{7}$ On peut en effet retrouver ce tableau dès 1973 dans le traité que consacre Broustet à la réadaptation des coronariens puis dans le «Guide du cœur et de l'effort physique» (Sourys- Rolland, op. cit.), édité par la FFC mais aussi dans le «Guide de la vie quotidienne du cardiaque - Tome 2, les maladies cardiovasculaires, ce qu'il faut savoir pour « mieux » vivre avec » édité aussi par la FFC en 1991 (Dir, SourysRolland et Manus) mais également dans de nombreux journaux de la presse grand public.

${ }^{8}$ Voir «Les dix commandements du sportif amateur» publiés par la FFC dans le Guide du cœur et de l'effort physique de 1986 déjà cité.
} 
être très largement diffusé dans l'espace public, les cardiologues s'imposant alors comme une corporation centrale concernant cette thématique à partir du milieu des années 1970 multipliant dans cette perspective les campagnes visant la promotion de l'APS auprès du grand public comme la semaine du cœur en 1974 ou les parcours du cœur en 1976. S'associant au secrétariat d'État à la Santé et au ministère du temps libre, de la jeunesse et des sports ainsi qu'au CFES, la FFC va également produire en 1985 la brochure «Sport-Cœur-Santé » largement diffusée et en collaboration avec le CFES, lancer en 1985 la campagne d'information sur les MCV « Bouge ton cœur » pour notamment véhiculer l'utilisation de l'APS comme facteur de santé publique (La santé de l'Homme, ${ }^{\circ} 259,1985$ ).

Cette mainmise de la FFC sur la question APS-santé est également révélée par le fait que d'autres acteurs, plus proches du milieu sportif, délivrent également des recommandations quelque peu différentes mais qui seront très peu diffusées en France. Ainsi, le Conseil de l'Europe par l'intermédiaire du Conseil de la Coopération Culturelle lance à partir du milieu des années 1960 une politique du « Sport Pour Tous » qui a pour objectif de promouvoir l'APS auprès du plus grand nombre et où l'argumentaire lié à la santé est très présent. Celle-ci débouchera en 1979 par la rédaction dela « Chartre Européenne du sport pour tous » adoptée par le conseil des ministres européens responsables du Sport (Conseil del'Europe,1979) qui inciteles politiques publiques nationales à s'engager dans ce processus. Dans ce cadre, le professeur Astrand, figure de la physiologie de l'exercice, réalise un rapport en 1969 travaillant les rapports entre APS et santé. Proches du mouvement sportif ${ }^{9}$, les recommandations d'Astrand sont quelque peu en décalage par rapport au discours médical dominant, alors même qu'elles s'adressent au même type de population, celui-ci privilégiant un « exercice assez violent, mais non maximal, pendant 2 à 5 min, suivi d'une période de repos ou d'exercice léger de même durée, puis d'exercices plus violents et ainsi de suite 3 à 5 fois » (Astrand, 1969). Contrairement aux recommandations délivrées par les médecins-cardiologues, les efforts continus de faible intensité ne sont pas ici particulièrement préconisés, préférant les exercices intermittents à intensité élevée. Ainsi, sans s'opposer sur les raisons sanitaires de promouvoir l'APS au plus grand nombre, les représentants du Sport pour Tous et les médecins-cardiologues de la FFC divergent quant aux efforts recommandés. Pour autant, bien que les acteurs s'inscrivant dans ce mouvement au niveau européen donnent des indications relativement précises en

${ }^{9}$ En France l'action du Sport pour Tous sera portée par le CNOSF et les fédérations sportives (Piard, 1974). termes d'effort et de pratiques, en France, celles-ci ne sont que faiblement relayées. Quand des recommandations apparaissent dans l'ensemble des documents parcourus, ce sont le plus souvent les indications rédigées par la FFC qui sont diffusées telles que les « dix commandements du sportif amateur $»^{10}$. La FFC s'installe alors comme l'acteur central de la période 1965-1985 sur la question de la lutte contre la sédentarité et incarne le discours légitime sur cette question ${ }^{11}$.

\section{Discussion}

Si la FFC s'installe comme un protagoniste de premier plan sur la question du sport-santé à cette période, se pose logiquement la question de l'intentionnalité des cardiologues dans la volonté de promouvoir l'APS au plus grand nombre. Quel intérêt la FFC avait-elle à encourager la prévention des MCV par l'APS dans le champ de la santé publique ? Notre matériel empirique ne nous permettant pas d'affirmer une réponse définitive, nous soumettons ici quelques hypothèses explicatives. Tout d'abord, en suivant la proposition de Merlaud [6] dans un autre contexte, l'AP comme thérapeutique semble moins controversée dans l'arène scientifique que les techniques médicamenteuses et fait relativement consensus à la fin des années 70 . D'autre part, cette période se caractérise également par un double mouvement de développement de la prévention dans une logique de critique du « tout curatif » [26] et par une volonté d'utilisation de thérapeutiques non-médicamenteuses dans la sphère médicale, pouvant potentiellement expliquer l'investissement de la FFC dans la promotion de l'APS comme outil de santé publique. Il est ainsi par exemple précisé dans un article concernant le stress comme facteur de risque des MCV dans La santé de l'Homme n ${ }^{\circ} 257$ en 1985 que : « [...] il pourrait guider le médecin vers une prescription autre qu'uniquement médicamenteuse ou diététique pour son patient à risque : relaxation, sport, auto-observation, prise en charge psychologique, analytique ou non, peuvent aussi être l'une des voies de la prévention des maladies cardiovasculaires ».

\footnotetext{
${ }^{10}$ Voir par exemple la brochure : « Le Sport bien-être en Aquitaine. Guide du Sport pour Tous » (Ministère de la Jeunesse et des sports, 1986).

${ }^{11}$ Pour une analyse précise et complémentaire des rapports entre les représentants Jeunesse et Sport et la Fédération Française de Cardiologie, nous renvoyons le lecteur à Radel [26] où l'auteur montre la mainmise de la Fédération dans l'organisation des parcours du cœur vis-à-vis des autres institutions investies dans le champ de la lutte contre la sédentarité.
} 
D'autre part, la FFC nous semble également s'installer comme un acteur de premier plan concernant le problème de la sédentarité à cette période dans la mesure où elle ancre pleinement son argumentaire dans le discours dominant sur la question et qu'elle contribue en retour à le renforcer. Bergeron [28], étudiant la mise à l'agenda politique de la question de la lutte contre l'alcoolisme indique que «qualifier un problème, ce n'est guère découvrir une réalité objective cachée. C'est plutôt présenter une réalité sous un jour particulier. Or, ces opérations de traduction ou de transcodage ne sont pas neutres. Elles participent à la désignation des experts légitimes. En définissant le problème, on contribue à définir les solutions. [...] L'expert construit le problème en même temps qu'il impose sa propre nécessité et celle de son expertise. Ce travail de qualification/requalification peut donc être perçu comme un moyen de s'octroyer une place dans le processus de décision publique ». La manière dont un problème public est qualifié n'est jamais neutre et participe à la désignation des acteurs légitimes ainsi que des solutions à mettre en place. Or, comme nous l'avons vu plus haut, à partir du milieu des années 1960, la question de la lutte contre la sédentarité est majoritairement cadrée par la sphère médicale afin de répondre au problème des MCV lui-même défini selon un paradigme épidémiologique où l'importance des facteurs de risque (dont le manque d'AP) est soulignée. Dès lors, dans la mesure où la FFC s'inscrit logiquement comme la communauté d'experts spécialistes des MCV, le fait que la question de l'AP en lien avec la santé soit publicisée très majoritairement dans le cadre de la prévention de ces pathologies offre la possibilité aux cardiologues, qui la saisissent, de s'inscrire comme les experts légitimes du problème. D'autre part, en affiliant totalement son argumentation à une approche épidémiologique et en publicisant la question de l'AP comme solution pour lutter contre le facteur de risque que représente la sédentarité, la FFC s'impose là encore comme l'acteur central et légitime sur la question. S'appuyant sur un raisonnement probabiliste, il est soutenu que la pratique d'AP permettrait de prévenir, de manière primaire et secondaire, le risque de développer une MCV (Demarais, 1986). Ainsi, la FFC s'ancre pleinement dans le discours légitime concernant la question de l'AP comme facteur de santé, et participe également à la diffusion et promotion de ce cadrage du problème.

Par ailleurs, si la question de l'AP comme facteur de santé pour le plus grand nombre subit une inflexion de sa trajectoire par un dé-confinement qui la porte sur le devant de la scène publique en lien avec la construction du problème des MCV durant la période 1960-1980, par la suite, la question de l'obésité qui va prendre une place importante en termes de santé publique dans les années 1990 et 2000 va également contribuer à une publicisation massive de cette cause, amenant à des bifurcations en termes de discours et de représentations sur cette question dans les sphères scientifiques, associatives, médiatiques et politiques qu'il s'agit d'investiguer.

Enfin, si nous avons travaillé à caractériser le processus de dé-confinement de cette question depuis l'arène scientifique vers des espaces publics, notamment dans la sphère de l'éducation à la santé, l'étude des médias généralistes et non pas spécialisés dans le domaine de la santé, reste à réaliser pour préciser ce mouvement. De plus, nous avons abordé la question de l'intentionnalité des acteurs et notamment de la FFC dans la promotion de l'APS comme outil de santé publique mais nous en sommes restés au stade de la formulation d'hypothèses. L'étude empirique spécifique de cette fédération semble ainsi nécessaire pour dépasser ce premier stade de réflexion.

Aucun conflit d'intérêt déclaré

\section{Références}

1. Rieu M. La santé par le sport : une longue histoire médicale. La revue pour l'histoire du CNRS. 2010;26:30-35.

2. Collinet $C$. Les grands courants d'éducation physique en France. Paris : PUF ; 2000. 280 p. (Pratiques corporelles).

3. Caby I, Blondel N. Les effets de l'activité physique sur la santé. In : Carlier G, éditeur. La santé. Paris : Éditions EPS ; 2008. pp. 29-46.

4. Bruant G. Le sport c'est la santé : itinéraire d'une représentation. In : Manidi M-J et Dafflon-Arvanitou I, éditeurs. Activité physique et santé. Paris : Masson ; 2000. pp. 10-17.

5. Radel A. 50 ans de campagnes d'éducation pour la santé. L'exemple de la lutte contre la sédentarité et de la promotion des modes de vie actifs (1960-2010) [Thèse de doctorat]. Toulouse : CLESCO, Université Paul Sabatier Toulouse III ; 2012. 473 p. [Visité le 07/12/2015]. En ligne : http://thesesups.ups-tlse.fr/1748/1/2012TOU30106.pdf.

6. Merlaud F. La médecine de l'obésité en France. Sociologie des engagements experts dans la normalisation de la corpulence [Thèse de doctorat]. Toulouse : CLESCO, Université Paul Sabatier Toulouse III ; 2012. 473 p. [Visité le 07/12/2015]. En ligne: http:// thesesups.ups-tlse.fr/2422/1/2014TOU30088.pdf.

7. Ferez $\mathrm{S}$, Thomas J. Sport et VIH. Un corps sous contrainte médicale. Paris : Téraèdre ; $2012.150 \mathrm{p}$.

8. Terret T, Perrin C. Activité physique et diabète en France : du rejet de la pratique à l'éducation du patient. Stadion. 2007;33(2):185-206.

9. Fuchs A. Les activités physiques et sportives dans le traitement de la mucoviscidose : enquête sur la qualité de vie d'un groupe d'enfants âgés de 8 à 13 ans [Thèse de doctorat]. Strasbourg (France) : Université de Strasbourg ; 2011. 450 p. 
10. Cazal J. Prévenir la maladie cardiovasculaire : socio-ethnologie du risque et de l'incitation sanitaire [Thèse de doctorat]. Toulouse : CLESCO, Université Paul Sabatier Toulouse III ; 2012.377 p. [Visité le 07/12/2015]. En ligne : http://thesesups.ups-tlse.fr/1727/1/ 2012 TOU30114.pdf.

11. Chateauraynaud F. Argumenter dans un champ de forces : Essai de balistique sociologique. Paris : Pétra ; 2011. 484 p. (Pragmatismes).

12. Gilbert C, Henry E. Comment se construisent les problèmes de santé publique. Paris : La Découverte ; 2009. 296 p. (Recherches).

13. Gusfield JR. The culture of public problems : drinking-driving and the symbolic order. Chicago (USA) : University of Chicago Press; 1980. $278 \mathrm{p}$.

14. Saint-Martin J, Travaillot Y, Labecq PA, Morales Y. L'œuvre du Dr Philippe Tissié : une croisade sociale en faveur de l'éducation physique (1888-1914). Bordeaux (France) : Presses Universitaires de Bordeaux; 2012. 216 p. (Regards croisés sur le sport).

15. Lebecq PA, Morales Y, Saint-Martin J, Travaillot Y. L'exercice et la santé : identité de la gymnastique volontaire en France depuis 1954. Lille : Le Manuscrit ; 2013. 337 p. (Sport Social Studies).

16. Giroux E. Origines de l'étude prospective de cohorte : épidémiologie cardiovasculaire américaine et étude de Framingham. Revue d'histoire des sciences. 2011;64(2):297-318. doi : 10.3917/ rhs.642.0297.

17. Soler L. Pluralité des paradigmes et dimensions de I'incommensurabilité en physique. In : Quidu M, éditeur. Innovations théoriques en STAPS et implications pratiques en EPS : les Sciences du sport en mouvement. Paris: L'Harmattan ; 2014. pp. 335-374. (Mouvements des savoirs).

18. Giroux E. Épidémiologie des facteurs de risque: Genèse d'une nouvelle approche de la maladie [Thèse de doctorat]. Paris : IHPST, Université Paris-1 ; 2006. [Visité le 07/12/2015]. En ligne : https:// halshs.archives-ouvertes.fr/halshs-00791927/.

19. Giroux E. Enquête de cohorte et analyse multivariée : une analyse épistémologique et historique du rôle fondateur de l'étude de Framingham. Rev Epidemiol Sante Publique. 2008;56(3):177-88.

20. Aronowitz RA. The Framingham heart study and the emergence of the risk factor approach to coronary heart disease, 1947-1970. Revue d'histoire des sciences. 2011;64(2):263-95.

21. Peretti-Watel P. Du recours au paradigme épidémiologique pour l'étude des conduites à risque. Revue française de sociologie. 2004;45(1):103-32.

22. Roussille B. Soixante ans d'éducation pour la santé. La Santé de I'Homme. 2002;302:14-20.

23. Beck U. La société du risque : sur la voie d'une autre modernité ? Paris : Aubier ; 2001. 521 p. (Champs essais).

24. Fassin D. Faire de la santé publique. Paris : EHESP ; 2005. 69 p.

25. Queval I.S'accomplir ou se dépasser : essai sur le sport contemporain. Paris : Gallimard ;2004.352 p. (Bibliothèque des sciences humaines).

26. Bossy T. Les différentes temporalités du changement: la mise sur agenda de l'obésité en France et au Royaume-Uni. In : Palier B, Surel Y, éditeurs. Quand les politiques changent : temporalités et niveaux de l'action publique. Paris : L'Harmattan ; 2010. pp. 145-82.

27. Radel A. Cardiologie et promotion de la santé par l'activité physique : approche historique d'une contribution fondatrice (19731986). Stadion. À paraître.

28. Bergeron H. Qualifier en politique : I'exemple du problème alcool. Santé Publique. 2008;20(4):341-52.

29. Van Der Maren JM. La recherche qualitative peut-elle être rigoureuse ? Cahier de recherche HEC.1996; 96 : 1-11.

\section{Annexe : Corpus empirique cité dans le texte}

- Astrand PO. Sport pour tous, exercice et santé. Strasbourg : Conseil de la coopération culturelle, Conseil de l'Europe ; 1969. 38 p.

- Aujoulat JP. Éditorial. La Santé de l'Homme. 1964;133:1-2.

- Aujoulat JP. Le cœur au cœur de la santé. La Santé de I'Homme. 1972;178:2-9.

- Breslow L, Buell P. Mortality from coronary heart disease and physical activity of work in California. J Chronic Dis. 1960;11(4):421-44.

Brondani JC. Influence de la pratique du judo sur le rachis en période de croissance [Thèse de médecine]. Paris : Université Paris VII, Faculté de médecine Lariboisière-Saint-Louis; 1974.

- Brown RG, Davidson LAG, McKeown T, Whitfield AGW. Coronary artery disease: influences affecting its incidence in males in the seventh decade. Lancet. 1957;270(7005):1073-7.

- Broustet JP. La Réadaptation des coronariens. Tour (France) : Sandoz éditions; 1973.375 p.

Broustet JP, Bricaud $\mathrm{H}$, Emeriau JP. Réadaptation fonctionnelle après un infarctus du myocarde. La Santé de l'Homme. 1976;205:24-28.

- Calo A. L'Athérome, maladie de civilisation. La Santé de l'Homme. 1960;113:48-49.

- Chapman J, Goerke L, Dixon W, Loveland D, Philips E. Measuring the risk of coronary heart disease in adult population groups: the clinical status of a population group in Los Angeles under observation for two to three years. Am J Pub Health. 1957;47:33-42.

- Comité pour le développement du sport (CDDS). European seminar on testing physical fitness: National Institute for Sport and Physical Educaion, Paris, 26-28 october 1978. Strasbourg : CDDS; 1979.

- CreffJ. Quelques réflexions à propos du sport et de la diététique. La santé de l'homme. 1966;143:13-16.

- Dawber TB, Kannel WB, Mc Namara PM. The prediction of coronary heart disease. Trans Assoc Life Insur Med Dir Am. 1964;47(70): 70-105.

Delore P. Tendances et besoins de l'éducation sanitaire. La Santé de I'Homme. 1960;113:3-5.

- Desmare P. Activités physique et rhumatismes. La Santé de l'Homme 1976;203:34-40.

Demarais Y. Sport de loisir et de santé : il faut d'abord lutter contre l'inactivité. In Souyris-Rolland A, éditeur. Guide du cœur et de l'effort physique. Paris : Edicardio ; 1986. pp. 26-27.

- Diaz R. Résultats pratique de l'entraînement physique à l'âge avancé. Actua. 1973;82(5):94-98.

- Duval H. De l'influence des efforts physiques et du surmenage sur la tuberculose pulmonaire. [Thèse de médecine]. 1950 ; Paris.

Froment R. Prophylaxie du rhumatisme articulaire aigu. La Santé de I'Homme. 1967;151:4-15.

- Hollmann W. Körperliches Training als Prävention von HerzKreislaufrankheiten. Stuttgart (D) : Hippokrates Verlag ; 1965.

Kahn JF. Retentissement médico-physiologique d'une saison de basket-ball [Thèse de médecine]. Nancy: Université de Nancy, faculté de médecine ; 1974.

- Karvonen MJ, Kihlberg J, Määttä J, Virkajrävi J. Longevity of champion skiers. Duodecim, 1956;72:893-903.

Katz LN. Physical fitness and coronary heart disease. Circulation. 1967;35:405-414.

- Keys A. Coronary heart disease in seven countries. Circulation. 1970 ; 41(4):186-95. 
- Kilbom A, Hartley L.H, Saltin B, Bjure J, Grimby G, Astand I. Physical training in sedentary middle aged and older men. Scand J Clin Lab Invest. 1969;24(4):315-44.

- Sur le terrain, bouge ton cœur, réseau accords-cœur. La santé de I'Homme. 1985;259:15.

- A comme à cran. La santé de l'Homme. 1985:257:32.

- LeclaincheX. Un fléau social méconnu : les maladies du cœur et des vaisseaux. La Santé de l'Homme. 1967;151:32-44.

- Longueville L, Melon JM, Wintrebert H, Hennape F. Le Vieillissement et le sport. Paris : Groupe d'étude du troisième âge sportif; 1965. $63 \mathrm{p}$.

- Milon H, Froment R. Prévention de l'athérosclérose et de l'infarctus du myocarde. La Santé de I'Homme. 1971;173:10-15.

- Ministère de la Jeunesse et des sports. Le Sport bien-être en Aquitaine. Guide du Sport pour Tous. Bordeaux : Edition Gironde ; 1986.

- Morris JN, Heady JA, Raffle PAB, Roberts CG, Parks JW. Coronary heart disease and physical activity of work. Lancet. 1953;2: $1111-1120$

- Organisation Mondiale de la Santé (OMS). Études de la morbidité et de la mortalité : tendances actuelles. Cahiers de la santé publique. 1967;27(209): $211 \mathrm{p}$.

- Perie H, Chignon JC, Dumas JP. Les activités physiques de l'enfant de 6 à 11 ans. La Santé de I'Homme 1973;183:8-14.

- Perrin A. La prévention des maladies cardiovasculaire. La Santé de I'Homme. 1967;133:17-33.
Piard C. Vers une nouvelle politique Sportive "Sport pour tous", dernière chance des fédérations. Paris : Amphora ; 1974. 114 p.

- Réville P. Sport Pour Tous, Les activités physiques et la prévention des maladies: faits et chiffres. Strasbourg: Conseil de l'Europe, Conseil de la coopération culturelle; 1970.46 p.

- Rook A. An investigation into the longevity of Cambridge sportsmen. Br Med J. 1954;1(4865):773-7.

- Saltin B, BlomqvistG, Mitchell JH, Johnson R, Wildenthal K, Chapman CB. Response to submaximal and maximal exercise after bedrest and training. A longitudinal study of adaptive changes in oxygen transport and body composition. Circulation. 1968;37/38: 1-78.

- Saltin B, Grimbly G. Physiological analysis of middle aged and old former athletes, comparison with still active athletes of the same ages. Circulation. 1968;38:1104-1115.

- Soisson JP. Activités physiques, sport et santé. Paris : La documentation Française ; 1980. 118 p.

- Souyris-Rolland A. Guide du cœur et de l'effort physique. $2^{\mathrm{e}}$ édition. Paris : Fédération de cardiologie; 1986.160 p.

- Souryis-Rolland A et Manus JM, Bruel A. Guide de la vie quotidienne du cardiaque - Tome 2, les maladies cardiovasculaires, ce qu'il faut savoir pour " mieux " vivre avec Paris : Edicardio ; 1991. 152 p.

- Varnauskas E, Bergman H, Houk P, Björntorp P. Hemodynamic effects of physical training in coronary patient. Lancet. $1966 ; 2(7453): 8-12$.

- Zarka M. Contribution à l'étude de l'électrocardiogramme du sportif [Thèse de médecine]. Paris : Université Paris 6, 1981. 76 p. 